\section{POTENT TUMOR ORGANOID INFILTRATION AND KILLING BY PBMC-DERIVED EFFECTOR CELLS}

${ }^{1}$ Frank Borriello*, 'Joshua Keegan, ${ }^{2} J a m e s$ Lederer. 'Alloplex Biotherapeutics, Inc., Woburn, MA, USA; ${ }^{2} B$ WH Harvard Medical School and Alloplex, Millis, MA, USA

Background Alloplex Biotherapeutics has developed a novel autologous cellular therapy for cancer that uses ENgineered Leukocyte ImmunoSTimulatory cell lines called ENLIST cells to activate and expand a heterogeneous population of tumor killing effector cells from human peripheral blood mononuclear cells (PBMCs). The 2-week manufacturing process from PBMCs consistently results 300-fold expansion of NK cells, CD8 + T cells, gamma/delta T cells, NKT cells and some CD4 $+\mathrm{T}$ cells, collectively called SUPLEXA therapeutic cells. SUPLEXA cells will be delivered back to cancer patients via intravenous administrations on a weekly schedule as an autologous adoptive cellular immunotherapy for cancer. In this study, we tested SUPLEXA cells developed from normal healthy volunteer PBMCs for their ability to infiltrate and kill patient-derived tumor organoids (PDO) as a pre-clinical assessment for potency against 2 different types of tumor organoids.

Methods Tumor organoids derived from colorectal cancer (CRC) or non-small cell lung carcinoma (NSCLC) patients were labeled with cell-trace red dye and plated at equal density in a 96-well plate. After 3 days culture, SUPLEXA cells were thawed $(82.8 \%$ viable), labeled with cell-trace violet dye, and added to PDO at 1:2 serial diluted numbers ranging from 2 million to 7,800 cells per well. Fluorescent images were captured at 24 hours after adding SUPLEXA cells to PDO models to measure PDO size, tumor infiltration, and PDO killing.
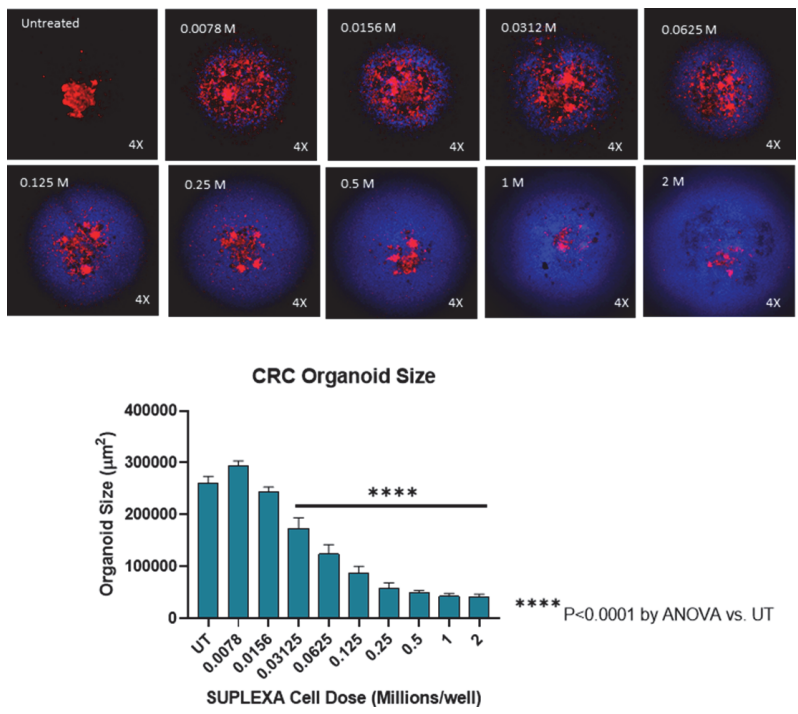

Abstract 164 Figure 1 CRC organoid infiltration and killing by SUPLEXA. A representative fluorescent image of CRC organoid killing with addition of increasing SUPLEXA cell numbers and a plot showing statistical analysis of 6 replicate wells for changes in CRC organoid size in relation to SUPLEXA cell number additions
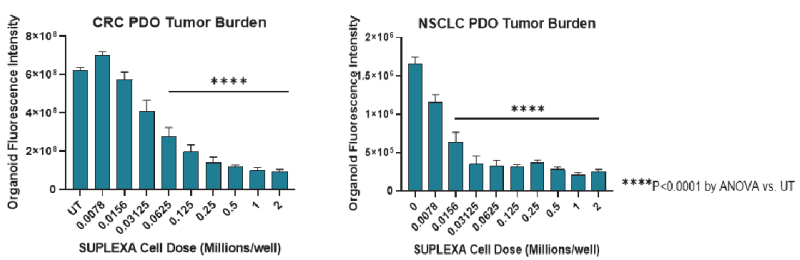

Abstract 164 Figure 2 Dose-dependent killing in CRC and NSCLC PDO models. CRC and NSCLC organoids were detected by total red fluorescence at 24 hours after adding the indicated numbers of SUPLEXA cells. Loss of red fluorescence after adding SUPLEXA is a measure of overall tumor cell killing/burden in organoids. Data is plotted as mean \pm SEM for $n=6$ replicates per group.

Results Adding SUPLEXA cells to PDO from CRC and NSCLC resulted in significant infiltration and killing of organoids by 24 hours as shown by the fluorescent images and the organoid size plot for the CRC PDO model (figure 1). Significant reduction in PDO size was observed by adding 31,240 SUPLEXA cells. Similar results were observed with the NSCLC PDO model with significant reduction in PDO size by adding 15,600 SUPLEXA cells. Obvious organoid infiltration was observed in both PDO models and organoid fluorescence was significantly reduced by addition of SUPLEXA cells in both PDO models to suggest that SUPLEXA cells were able to reduce tumor burden (figure 2).

Conclusions SUPLEXA cells infiltrated and killed tumor cells in patient-derived organoids within 24 hours of culture at low cell concentrations indicating potent tumor killing activity. The observed activity in both colorectal and lung cancer organoid models support broad anti-tumor killing activity by SUPLEXA. These results provide further evidence that PBMCs from cancer patients can be activated and expanded by our approach as a novel autologous cellular immunotherapy for cancer.

http://dx.doi.org/10.1136/jitc-2021-SITC2021.164 